\title{
Management of Respiratory Foreign Body- A Twelve Years Experience with 382 Cases
}

\author{
GOLAM MOHIUDDIN AKBAR CHOWDHURY ${ }^{1}$, AKM AKRAMUL HAQUE $^{1}$, ZILLUR RAHMAN ${ }^{1}$, ARM \\ LUTHFUL KABIR ${ }^{2}$, RUHUL AMIN ${ }^{3}$, NAWAB TAHSIN UDDIN ${ }^{1}$
}

\begin{abstract}
Objectives: To evaluate the clinico-demographic profile and management of foreign body (FB) aspiration in hospitalized patients.

Methodology: This was a retrospective study conducted at the National Institute of Diseases of the Chest and Heart (NIDCH), Dhaka during the period of October 1994 to February 2006. Medical records were used for collection of cases. The diagnosis of FB aspiration was made from documented presenting features, physical findings, investigations of chest radiology, CT scan whenever done and management outcome with rigid bronchoscopy and surgical interventions like bronchotomy or resection surgery.
\end{abstract}

Results: Most of the patients 374 (97.5\%) were children up to 15 years of age with the peak age of 1-10 years (81.3\%). Males were affected more (63\%) than females (37\%). The majority of the patients (96\%) were from poor socio-economic status. Most of the patients 297 (77.7\%) presented early (within 7 days of aspiration) with cough and respiratory distress. There was late (after 7 days up to 12 years) presentation in 85 (22.2\%) cases with respiratory complications. FB was found to be radio-opaque in $122(31.9 \%)$ cases on chest imaging. Rigid bronchoscopy was attempted in all cases initially and it was successful to remove $F B$ in $99.6 \%$ cases in patients who presented early. But it failed to remove the FB in 49 (57.6\%) of cases when the patients presented late. Most of the FBs were of natural in origin 247 (64.6\%) and the number of artificial FBs was less 135 (35.3\%). FBs found impacted more on the right bronchial tree in 230 (60.2\%), followed by left side in 121 (31.6\%) and central airway in 31 (8.1\%) cases. In failed cases, bronchotomy, lobectomy and pneumonectomy were the other modalities (57.6\%) of management.

Conclusion: Children of the age group 1-10 years were found most vulnerable for $F B$ aspiration. FB of natural origin were common in our situation. FBs were impacted mostly on the right side. Removal of FB by rigid bronchoscopy was excellent when the patients presented early. Other difficult modalities of management like bronchotomy, lobectomy and pneumonectomy were needed in cases of late presentation.

\section{Introduction}

In our country the FB in the airway is a common problem especially in the field of thoracic Surgery and paediatric medicine. FB aspiration is mostly a problem of children ${ }^{1}$. Usual victims are children of 6 months to

1. Department of Thoracic Surgery, National Institute of Diseases of the Chest and Hospital (NIDCH), Mohakhali, Dhaka

2. Faculty of Pediatrics, Institute of Child and Mother Health (ICMH), Dhaka

3. Dhaka Shishu Hospital, Dhaka

Correspondence: Dr. Golam Mohiuddin Akbar Chowdhury
5 years of age, because of their curious nature, strong oral tendency and lack of molar teeth. Mode of aspiration is always accidental. This results in emergency situations many a times. Presentation of FB aspiration may be of three types: (1) a previously healthy child has sudden choking with cough and wheezing while playing with small objects or eating things ${ }^{2}$. The diagnosis is obvious in such cases. (2) The presentation may be due to development of complications of FB aspiration like recurrent pneumonia in the same lobe following a history of FB 
aspiration and responding briefly with antibiotic therapy $^{3,4}$, slight wheeze ${ }^{5}$, bronchiectasis ${ }^{1,6}$ pneumothorax and pleural empyema ${ }^{7}$. (3) The FB aspiration even may be asymptomatic and is a rare event $^{8}$. The classical physical findings are the clinical triad of cough, wheezing and diminished or absent breath sounds ${ }^{9}$.

Though only $10 \%$ of the aspirated objects are radiopaque but chest radiology is an important investigation for suspected foreign body aspiration as obstructive emphysema is seen in $80 \%$ of infants and obstructive atelectasis in $20 \%$ cases $^{10}$. Other helpful investigations are chest film taken during inspiration is compared with another film taken after forced expiration, fluoroscopy, CT scan and rigid bronchoscopy. Rigid bronchoscopy is mandatory if there is a convincing history of foreign body aspiration, regardless of radiographic findings 2,10 . In case of late complicated cases it may need bronchotomy or resection of involved lung which undergoes ii-reversible parenchymal destruction like bronchiectasis or organizing pneumonia ${ }^{11,12}$. We are sharing our twelve years experience of managing foreign body aspiration in all ages of population at NIDCH.

\section{Materials and Methods}

This is a retrospective study conducted in the NIDCH during the period of October'94 to February'06.

Medical record files of admitted patients managed for FB aspiration were scrutinized for history, physical features, radiology and CT scan of lungs (in selected cases) features. Rigid bronchoscopy was attempted universally and surgical interventions whenever needed.

Patients were categorized as early or late presenters. Presentation within one week of aspiration was noted as 'early' and after that it was labeled as 'late' case. All the information regarding history, physical findings, chest radiology and imaging, nature of foreign body, treatment protocol, operative findings and follow up were documented in a structured data sheet.

\section{Results}

Sociodemographic features: Total number of cases was 382 during the period of study. The age range was 2 months to 72 years with 373 (97.6\%) children up to 15 years of age and the peak age of children was 1-5 years 192 (50.2\%). Males were affected more $(53.1 \%)$ than the female patients (46.8\%). Most of the patients (96\%) were from poor socioeconomic background.
Presentations: The patients (77.7\%) who presented early (within one week of aspiration) had cough and respiratory distress. The symptoms and sign varied in accordance to the types of FB, its size, shape and the site and degree of obstruction of the airway. Physical findings were decreased breath sound and localized wheeze in case of partial obstruction of airway. The late presenters (after one week) were 85 (22.2\%) was due to initial apparent improvement of cough and respiratory distress with medication and the foreign body being radiolucent on chest radiology. Tissue reaction occured and distal occlusion supervene. These patients presented with repeated attacks of pulmonary infections, features of bronchiectasis or fibrosis depending upon the degree and site of obstruction.

Investigations: X-Ray chest was done in all cases and CT scan was done in selected cases (15.1\%) cases. Radio-opaque FBs were seen in 122 (31.9\%) cases (examples in figure-1a and $1 \mathrm{~b}$ ).

Treatment: All patients who presented both early and late were initially subjected to rigid bronchoscopy. Endoscopic removal was successful in 332 (86.9\%) cases and in most of the early presenters (99.6\%). Rigid bronchoscopy could remove 36 (42.7\%) of 85 late presenting cases. Rigid bronchoscopy failed to remove FB in 50 (13.0\%) cases. Bronchotomy, lobectomy and pneumonectomy were other options for further management. Twenty three patients were treated with bronchotomy (examples in figure-2a and 2b) (right lower lobe 20, left lower lobe 2, lingula 1), 18 patients required lobectomy for lobar brocchiectasis (right lower lobe 14, left lower lobe 4) and 9 patients remained undiagnosed until pneumonectomy was performed (right side 3, left side 6). The management of FB aspiration is summerised in Table-1. FBs were located in right bronchial tree in $230(60.2 \%)$ cases, left bronchial tree in 121 (31.6\%) cases and in trachea 31 (8.1\%) cases. The removed FBs were of different types: in 247 (64.6\%) cases FBs were of organic origin like fruit seeds and peels, nuts, small fishes etc. and in $135(35.3 \%)$ cases, the FBs were of inorganic nature like pieces of toys, needles, pen ends, ornaments, any small household objects etc. (Figure-3). 


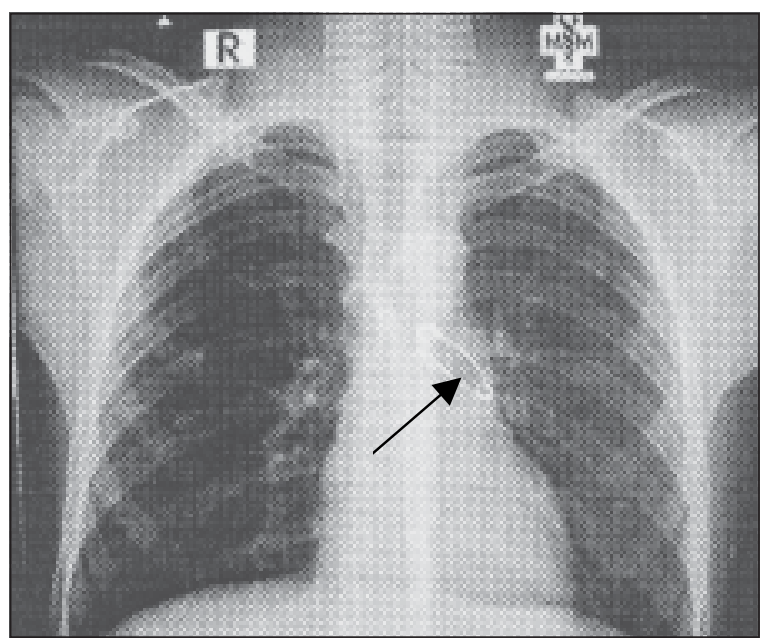

Fig.-1a: Foreign body in left principal bronchus in frontal projection

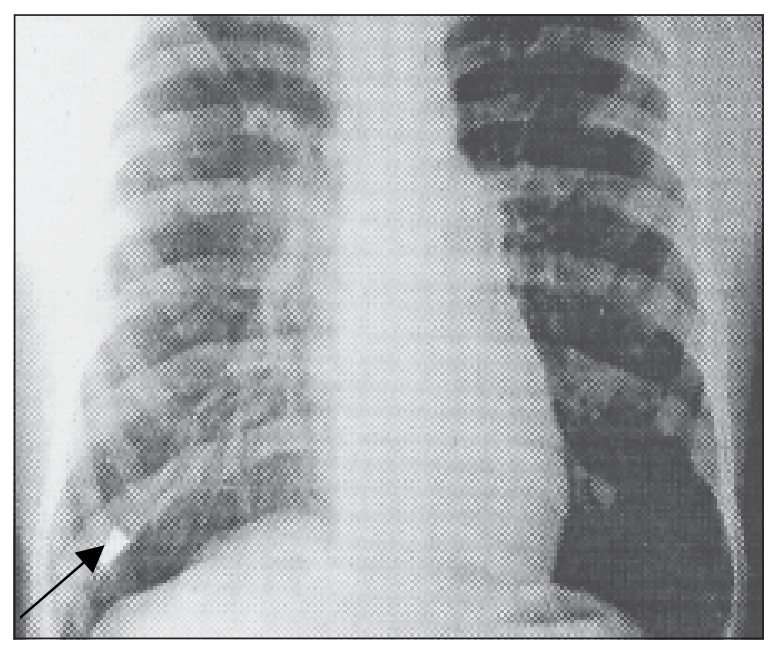

Fig.-2a: Foreign body in right distal airway

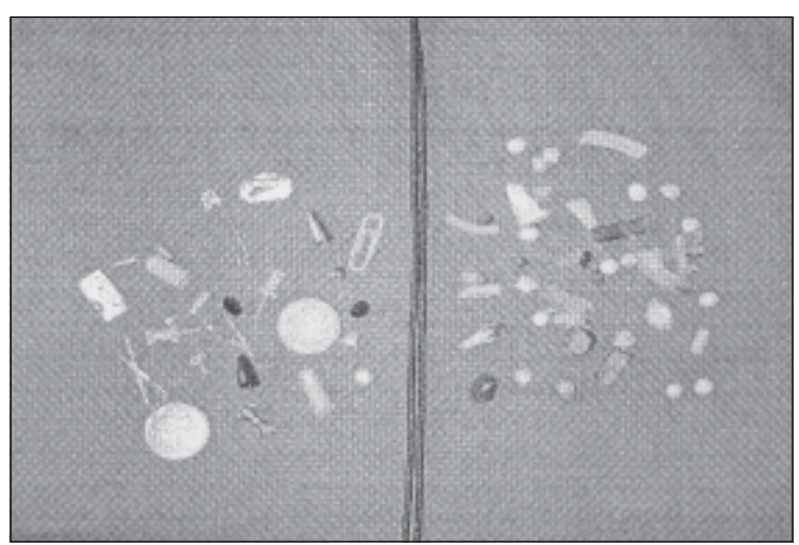

Fig.-3: Showing replica of foreign bodies of different natures

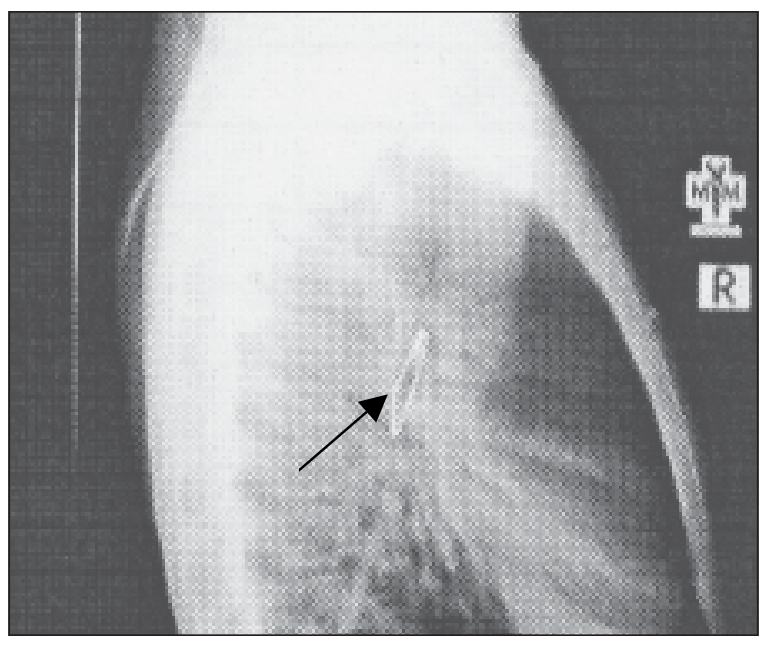

Fig.-1b: Foreign body in left principal bronchus on latral projection.

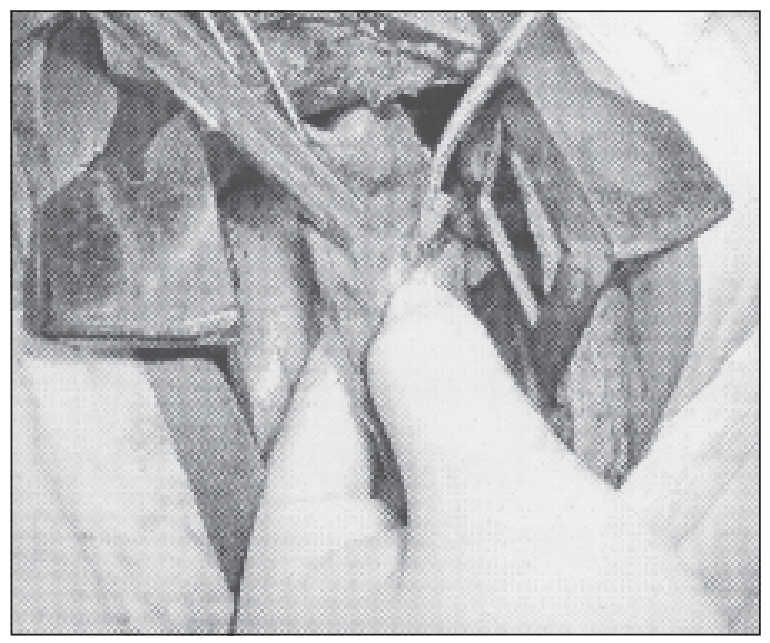

Fig.-2b: Foreign body being removed by bronchotomy

\section{Table-I}

Management of patients with FBs in airway presenting early and late $(n=382)$

\begin{tabular}{lccc}
\hline Management & $\begin{array}{c}\text { Total } \\
\text { No }\end{array}$ & $\begin{array}{c}\text { Early } \\
\text { No (\%) }\end{array}$ & $\begin{array}{c}\text { Late } \\
\text { No (\%) }\end{array}$ \\
\hline Endoscopic removal & 332 & $296(89.2)$ & $36(10.8)$ \\
Bronchotomy & 23 & $01(4.3)$ & $22(95.7)$ \\
Lobectomy & 18 & 00 & $18(100)$ \\
Pneumonectomy & 09 & 00 & $09(100)$ \\
\hline
\end{tabular}

Follow up: Every patient was observed for next 2472 hours for persistence of symptoms. Nine cases underwent re-bronchoscopy and retained foreign bodies were removed. Follow up continued up to 3 
years; though most of the cases did not return for follow up. The objective of follow up was to look for any clinical and radiological evidences for development of bronchial stenosis. One patient of late case who underwent bronchotomy, developed bronchial stenosis in right lower lobe bronchus treated with boogie dilatation. Subsequent follow up showed good result.

\section{Discussion}

This large study for a long duration on FB aspiration has highlighted one spectrum of accidental problem particularly in children. This is another report on FB aspiration of all ages but particularly in children after the report of Kabir in the year $2003^{1}$. The diagnosis of FB aspiration was made from documented history, physical features, radiological and imaging features and later confirmed after removal of FB either by rigid bronchoscopy or intervention surgery viz. bronchotomy or lobectomy for damaged lungs due to FB aspiration.

Most of the victims of FB aspiration were children up to 15 years of age with the peak age being 1 to 10 years. Children of younger age are the real victims of foreign body aspiration as observed in other studies $^{13,14}$.

In present study, the incidence of FB aspiration was more in boys. Similar observation was obtained in studies conducted by Tang FL et $\mathrm{al}^{13}$ where out of 304 children, 208 were boys and in another study, out of 663 cases in, 402 were boys ${ }^{15}$. Boys being more active, curious and naughty making them vulnerable to other accidents and emergencies like drowning ${ }^{16}$.

Aspirated FB was mostly organic (64.6\%) in this study. This was also corroborated by other Studies ${ }^{14}$ where $95 \%$ were of organic origin and $89.9 \%$ of total 1887 cases were also of organic types ${ }^{17}$. The more prevalence of organic FBs might be due to abundance of seasonal fruits having peels and seeds in our country.

Study conducted with 400 cases of FB aspiration by $\mathrm{Mu} \mathrm{L}$ et $\mathrm{al}^{14}$ showed most common presenting symptoms were cough, wheeze, dyspnoea and hoarseness of voice, decreased air entry, ronchi on the corresponding site. Study conducted by Zerella JT et al 18 where 265 children with history of early FB aspiration showed 228 cases had initial choking sensation following aspiration. The patients who presented early in our series had cough and respiratory distress.
Saquib Mallick M et al ${ }^{19}$ in their study with 128 cases showed that 17 out of 28 patients who presented late (after 1 month), developed complications including pneumonia, bronchiectasis and broncho-oesophageal fistula. In the present study, late FB aspiration cases had repeated attacks of cough, wheeze and lung infection. Radiologically, there were collapse, consolidation, features of bronchiectasis involving whole lung or depending on the site of involvement.

Though most of the FB were organic in nature and are not radiopaque, history was the mainstay of diagnosis. In this series, radio-opaque FB was 122 (31.9\%). Radiopaque FBs are found in less number of cases as observed in other study ${ }^{10}$ where only $10 \%$ cases were radiopaque. Other features in radiology were atelectasis, bronchiectasis, recurrent pneumonia, or fibrosis.

Rigid bronchoscopy was done in all cases as it is the gold standard for removing tracheobronchial foreign bodies in the cases of infants and children ${ }^{20}$. Successful removal was in 332 (86.9\%) cases. Surgical interventions (bronchotomy, lobectomy and pncumonectomy) were required in 50 (13.0\%) cases. Sirmali $\mathrm{M}$ et $\mathrm{al}^{21}$ studied 220 cases of $\mathrm{FB}$ aspiration and rigid bronchoscopy was tried in all cases. FBs were removed successfully from 209 (95\%) cases. Bronchotomy and resection surgery were needed in remaining 11 (5\%) cases.

Endoscopic removal showed excellent results without any residual effect, especially in early presented FB aspiration. In late cases, it required prolong antibiotics and other supportive therapy and follow up. Patients who needed bronchotomy also showed very good outcome except one case who presented after 6 months with little degree of stenosis diagnosed endoscopically and finally treated with endoscopic dilatation. On long term follow up, no permanent residual effect on lung tissue was noticed. Patients who underwent lobectomy also showed promising results in terms of relieving symptoms of repeated infection which is a feature of bronchiectasis. In cases of pneumonectorny, the results were also good in respect to improvement of symptoms due to pulmonary infection. But, 2 patients developed post operative space infection and were treated accordingly.

\section{Conclusion}

FB in airway is a serious pulmonary problem. Children are the main victims of $\mathrm{FB}$ aspiration. Rigid 
bronchoscopy is the mainstay of therapy for successful extraction of FBs in patients who presented early. There is fair chance of failure to remove FB with rigid bronchoscopy in late cases and surgical interventions (bronchotomy, lobectomy or pneumonectomy) are the main options for management in these case.

\section{References}

1. Kabir ARML, Ahmed F, Chowdhury GMA, Amin $\mathrm{R}$, Hossain $\mathrm{H}$. Foreign Body Aspiration in Children: Reports of 2 cases. Bang J Child Health 2003; 27: 64-66.

2. Somu N, Thangavelu S, Subramanyam L. Foreign body aspiration. In: Somu N, Subramanyam L, editors. Essential of Pediatric Pulmonology. Siva \& Co 1996; P. 59-62.

3. Minlar DA, Hull D. editors. In: Hospital Pediatrics. Churchill Livingstone 1998.

4. Boat TF. Pulmonary hemorrhage and hemoptysis. In. Chernick V, Boat TF, Kendig EL, editors. Kendig's disorder of the respiratory tract in children. WB Saunders Company 1998; P. 623-33.

5. Freiman MA, McMurray JS. Unique presentation of a bronchial foreign body in an asymptomatic child. Ann Oto Rhinol Laryngol 2001; 110: 49597.

6. Bertolani MF, Marotti F, Bergamini BM, Pellegrino M, Balli R, Calandra Buonaura PL. Extraction of a rubber bullet from a bronchus after one year: complete resolution of chronic pulmonary damage. Chest 1999; 115: 1210-13.

7. Amman FF, Faris KT, Mahafza TM. Inhalation of wild barley into the airways: two different outcomes. Saudi Med J 2000; 21: 468-70.

8. Aytac A, Yudaknl Y, Ikizler C. Inhalation of foreign bodies in children. J Thorac Cardiovasc Surg 1977; 74: 145-51.

9. Wiseman NE. The diagnosis of foreign body aspiration in childhood. J Pediatr Surg 1984; 19: 531-35.

10. Kirks DA. Practical Pediatric Imaging: Diagnostic Radiology in Infants and Children. Boston: Little Brown; 1984.
11. Hilmanet A1, Kane GC, Sloane PJ, McComb B. Missed foreign body in an adult. Respir Med 1994; 88: 551-54.

12. Henselmans JLM, Schramel FMNH, Sutedja G, Postmus PE. Acute necrotising pneumonia 16 years after aspiration of a conifer branch. Respir Med 1995; 89: 139-41.

13. Tang FL, Chen MZ, Zou CC, Zhao YZ. Fibrobronchoscopic treatment of foreign body aspiration in children: an experience of 5 years in Hangzhou city, China. Pediatr Surg 2006; 41:1-5.

14. $\mathrm{Mu} L$, , He P, Sun D. Inhalation of foreign bodies in Chinese children: A review of 400 cases. Laryngoscope 1991; 101: 657-60.

15. Ciftci AO, Bingol-Kologlu M, Sencak ME, Tanyel FC, Buvukpamukcu N. Bronchoscopy for evaluation of foreign body aspiration in children. J Pediatr Surg 2003; 38: 1170-76.

16. Rahman A, Rahman F, Shafinaz S, Linnan M. Bangladesh Health and Injury Survey, Report on Children. ICMH \& UNICEF, Bangladesh; 2005.

17. Avdogan LB, Tuncer U, Soylu L, Kiroglu M, Ozsahinoglu C. Rigid bronchoscopy for the suspicion of foreign body in the airway. Int J Pediatr Otorhinolaryngol 2006; 70: 823-28.

18. Zerella JT, Dimler M, McGill LC, Pippus KJ. Foreign body aspiration in children: value of radiography and complications of bronchoscopy. J Pediatr Surg 1998; 33: 1651-54.

19. Saquib Mallick M, Rauf Khan A, Al-Bassam A. Late presentation of tracheobronchial foreign body aspiration in children. J Trop Pediatr 2005; 551: 145-48.

20. Cotton RT. Foreign body aspiration. In: Chernich $\mathrm{V}$, Boat TF, Kendig EL, editors. Kendig's disorder of the respiratory tract in children. WB Saunders Company; 1998. P. 601-07.

21. Sirmali M, Turut $H$, Kisacik E, Findik G, Kava S, Tastape $\mathrm{L}$. The relationship between time of admittance and complications in paediatric tracheobronchial foreign body aspiration. Acta Chir Belg 2005; 105: 631-34 